\title{
An in computo investigation of the Landau-Kleffner syndrome
}

Maxime Bonjean, Christophe Phillips, Thien Thanh Dang Vu, Rodolphe Sepulchre, and Pierre Maquet

\begin{abstract}
We describe a computational model of the thalamus and the cortex able to reproduce some essential epileptiform features commonly observed in the LandauKleffner syndrome. Investigation with this realistic model leads us to the formulation of a cellular mechanism that could be responsible for the epileptic discharges occuring with this severe syndrome. Understanding this mechanism is of prime importance for developing new therapeutical strategies.
\end{abstract}

\section{INTRODUCTION}

Epilepsies are one of the most common neurologic disorders, with a prevalence of about $1 \%$ of the world's population, that manifests in diverse ways (Laidlow et al., 1993). There are numerous seizure types and numerous mechanisms by which the brain generates seizures. The two hallmarks of seizure generation are hyperexcitability of neurons and hypersynchrony of neural circuits (Stafstrom, 2006).

Among them, Landau-Kleffner syndrome (LKS) is a rare but severe disease, affecting children between 3 and 10 years of age, characterized by deterioration of one or more cognitive functions, behavioral disturbances, spike and spike-wave EEG discharges which increase during slow wave sleep, and epileptic seizures (Maquet et al., 1995).

LKS is a form of non-lesional age-related focal epilepsy which was first described over 50 years ago by Landau \& Kleffner, using clinical and EEG criteria, where aphasia represents the main neuropsychological deficit (Landau \& Kleffner, 1957). Most often, the symptoms consist of difficulties in comprehension due to an auditory agnosia, with rapid reduction of spontaneous oral language. Aphasia is usually associated

Manuscript received February 23, 2007; revised June 5th, 2007. This paper presents research results of the Belgian Network DYSCO (Dynamical Systems, Control, and Optimization), funded by the Interuniversity Attraction Poles Programme, initiated by the Belgian State, Science Policy Office. The scientific responsibility rests with its author(s).

M. Bonjean, IEEE Member, is with the Cyclotron Medical Research Center, School of Medicine, and with the Systems and Modeling Research Unit, School of Engineering, University of Liege, 4000 Liege, Belgium m.bonjean@ulg.ac.be

C. Phillips is with the Cyclotron Medical Research Center, University of Liege, Belgium

R. Sepulchre is the head of the Systems and Modeling Research Unit, School of Engineering, University of Liege, Belgium

P. Maquet \& T.T. Dang Vu are with the Cyclotron Medical Research Center and with the Department of Neurology, University of Liege, Belgium. with behavioral abnormalities such as attention deficit and hyperactivity. The waking EEG shows focal, multifocal or bilateral spike-wave discharges, the topography of which fluctuates with time (Maquet et al., 1995). These spike-wave discharges increase during slow-wave sleep and may become continuous. Occasional epileptic seizures (partial or generalized) occur in $70 \%$ of patients (Beaumanoir, 1992).

LKS involves deterioration of cognitive functions that were previously normally acquired, and may partially regress before the end of adolescence (Mantovani \& Landau, 1980). The epileptic seizures observed are partial motor seizures, atypical absences, and generalized tonic-clonic seizures. The EEG abnormalities consist of spike-and-wave discharges with fluctuating topography, the frequency of which is significantly increased during slow-wave sleep.

Although the clinical and EEG features of this syndrome are documented, understanding of the aetiology and pathogenesis of this affection still remains fragmentary. Likewise, the interaction between epileptiform abnormalities and cognitive dysfunction is not fully understood. In this paper, we describe the development of a realistic model and the use of computer simulations of layers thalamocortical circuits with the aim of simulating some aspects of the epileptiform behavior of this pathology. Without leading to definitive conclusions, our results could however shed new light on some interesting pathophysiological mechanisms with this in computo approach, giving the right direction of future research for this serious affection.

\section{METHODS}

A thalamocortical network composed of a total of 3,600 cells was simulated. The cells were distributed into four different layers: the nuclei of the thalamus were composed of one layer for the thalamocortical relay (TC) neurons and one layer for the thalamic reticular (nRT) neurons. The two last layers were to describe pyramidal excitatory (PY) neurons (layer V) and inhibitory interneurons (IN), respectively. The ratio between PY and IN were 4:1, even if the exact proportions seem not relevant for the results. Each neuron has afferences and efferences 
through biologically plausible stochastic distributions. Heterogenity in a same layer was achieved though a random distribution of parameters.

\section{A. Model neurons}

Given computational limitations, large realistic networks are not praticable with detailed morphologically reconstructed neurons. Because simulation of such a network typically requires a model cell with a minimal number of compartments, we used for all of our model cells somatic and dendritic compartments (Pinsky \& Rinzel, 1994). Even if these models are not as accurate as the real cells in simulating the integration of synaptic inputs in the dendritic compartments and the resulting flow of current into the soma, they have been proven to be sufficient to capture the relevant electrophysiological aspects.

Each class of neurons has the currents $I_{N a}$ and $I_{K, d r}$, responsible for the generation of action potentials, located in the soma, as well as a leak current $I_{l}$. Thalamocortical relay (TC) cells have in addition $I_{T}$, lowthreshold $\mathrm{Ca}$-current, $\mathrm{I}_{L}$, high-threshold $\mathrm{Ca}$-current, a $\mathrm{Ca}^{2+}$-dependent $\mathrm{K}^{+}$-current $\mathrm{I}_{K[\mathrm{Ca}]}$, a non-inactivating $\mathrm{Na}^{+}$-current $I_{N a, p}$, and $I_{h}$, hyperpolarization-activated non-specific current. Thalamic reticular (nRT) cells have $I_{T s}$, same as $I_{T}$ but with a slower dynamics, $I_{L}, I_{K[\mathrm{Ca}]}, I_{C A N}$, and $I_{N a, p}$. Cortical pyramidal (PY) cells have in their soma a fast $K^{+}$A-current $I_{A}$, a non-inactivating slow $K^{+}$-current $I_{K s}$, and a $\mathrm{Na}^{+}$dependent $K^{+}$-current $I_{K[N a]}$. PY dendrites contain a high-threshold $\mathrm{Ca}^{2+}$-current $\mathrm{I}_{\mathrm{Ca}}$, a $\mathrm{Ca}^{2+}$-dependent $\mathrm{K}^{+}$current $I_{K[\mathrm{Ca}]}$, a non-inactivating $\mathrm{Na}^{+}$-current $I_{\mathrm{Na}, \mathrm{p}}$, and an hyperpolarization-activated inward rectifier $K^{+}$current $I_{A R}$ (non-inactivating). Cortical interneurons (IN) were modeled with the spiking currents $I_{N a}$ and $I_{K}$, into a single compartment, as well as the leak current $I_{l}$ (Shepherd, 2001).

Each compartment $i$ composing the cell model was described by a nonlinear differential equation accounting for the dynamics of their voltage $V_{i}$,

$$
C_{m} A_{i} \dot{V}_{i}=-g_{l}\left(V_{i}-E_{l}\right)-A_{i} \sum_{j} I_{j i}^{i n t}-\sum_{k} I_{k i}^{s y n}
$$

where $C_{m}=1 F / \mathrm{cm}^{2}$ is the specific membrane capacitance, $A_{i}$ is the compartment area, $g_{l}$ is the leakage conductance, and $E_{l}$ is the leakage reversal potential. In the case of more than a single compartment, Eq. (1) has a new right term accounting for the electrical coupling between compartments $i$ and $k$, i.e., $-g_{i k}\left(V_{i}-V_{k}\right)$. Intrinsic currents followed Hodgkin-Huxley-like kinetics,

$$
I_{j i}^{i n t}=\bar{g}_{j} m_{j}^{M} h_{j}^{N}\left(V_{i}-E_{j}\right)
$$

where the current is expressed as the product of the maximal specific conductance $\bar{g}_{j}$, activation $m_{j}$ and inactivation $h_{j}$ variables, and the difference between the membrane potential $V_{i}$, and the reversal potential $E_{j}$. Activation and inactivation gates follow the single twostate kinetic scheme (Destexhe \& Sejnowski, 2001). Expanding Eq. 1 with the exact ionic currents, we obtain for PY cells,

$$
\begin{aligned}
C_{m} A_{S} \dot{V}_{S}= & -A_{S}\left(I_{l}+I_{N a}+I_{K}+I_{A}+I_{K s}+I_{K[N a]}\right) \\
& -I_{s y n, S}-g_{s d}\left(V_{S}-V_{d}\right), \\
C_{m} A_{d} \dot{V}_{d}= & -A_{d}\left(I_{C a}+I_{K[C a]}+I_{N a, p}+I_{A R}\right) \\
& -I_{s y n, d}-g_{s d}\left(V_{d}-V_{S}\right) ;
\end{aligned}
$$

for IN cells,

$$
C_{m} A=-A\left(I_{l}+I_{N a}+I_{K}\right)-I_{s y n}
$$

for TC cells,

$$
\begin{aligned}
C_{m} A_{S} \dot{V}_{S}= & -A_{S}\left(I_{l}+I_{N a}+I_{K}+I_{T}+I_{L}+I_{h}\right) \\
& -I_{s y n, S}-g_{s d}\left(V_{S}-V_{d}\right) \\
C_{m} A_{d} \dot{V}_{d}= & -A_{d}\left(I_{K[C a]}+I_{N a, p}\right) \\
& -I_{s y n, d}-g_{s d}\left(V_{d}-V_{S}\right)
\end{aligned}
$$

and for nRT cells,

$$
\begin{aligned}
C_{m} A_{S} \dot{V}_{S}= & -A_{S}\left(I_{l}+I_{N a}+I_{K}+I_{T s}+I_{L}\right) \\
& -I_{s y n, S}-g_{s d}\left(V_{S}-V_{d}\right), \\
C_{m} A_{d} \dot{V}_{d}= & -A_{d}\left(I_{K[C a]}+I_{N a, p}+I_{C A N}\right) \\
& -I_{s y n, d}-g_{s d}\left(V_{d}-V_{S}\right) .
\end{aligned}
$$

\section{B. Model ion channels}

All ion channels are modeled following the HodgkinHuxley formalism (Hodgkin \& Huxley, 1952), with gating variables $x$ governed by the first-order kinetics equation,

$$
\begin{aligned}
\dot{x} & =\Phi\left[\alpha_{x}(V)(1-x)-\beta_{x}(V) x\right] \\
& =\frac{\Phi}{\tau_{x}(V)}\left(x_{\infty}(V)-x\right),
\end{aligned}
$$

$\Phi$ being the temperature factor. Accurate description of the current parameters were taken from the literature (Wang, 1998; Shepherd, 2001).

\section{Model synapses and connectivity}

Both excitatory (AMPA and NMDA) and inhibitory $\left(\mathrm{GABA}_{A}\right.$ and $\left.\mathrm{GABA}_{B}\right)$ synapses were modeled. The synaptic currents followed

$$
I_{k i}^{s y n}=\bar{g}_{k i} m_{k i}\left(V_{i}-E_{k i}\right),
$$

where subscript $k i$ indicates the synaptic contact from neuron $k$ to neuron $i, \bar{g}_{k i}$ is the maximal conductance, and $E_{k i}$ is the reversal potential of the postsynaptic current. The fraction of open receptors $m_{k i}$ for fast postsynaptic currents (AMPA, $\mathrm{GABA}_{A}$ ) was modeled by a two-state kinetic scheme (Destexhe et al., 1994), whereas slow postsynaptic currents NMDA and $\mathrm{GABA}_{B}$ receptors had a more complex activation scheme based on the kinetics of G-proteins (Destexhe \& Sejnowski, 
1995).

The corticothalamic feedback loop was modeled. Intrathalamic and intracortical connections are local and topographically organized, following a stochastic distribution. Neurons can make multiple contacts onto the same target. The probability that they are connected in each direction is decided by a normal Gaussian probability distribution,

$$
P(x)=\frac{\exp \left(-\frac{x^{2}}{2 \sigma^{2}}\right)}{\sqrt{2 \pi \sigma^{2}}},
$$

taken at discrete points with $x=n \mathrm{~d} x$ values, where $n$ is the discrete index to the target neuron with respect to the efferent neuron and $\mathrm{d} x$ is the separation distance between neurons. For inhibitory connections, a normal Gaussian probability distribution is also used with a small standard deviation. Fig. 1 illustrates the 4-layer model and the various synaptic interconnections.

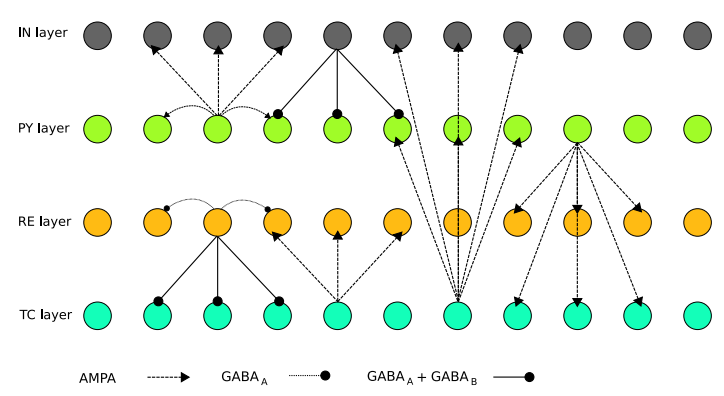

Fig. 1. Schematic view of the model. Neurons are divided into 4 different layers, each corresponding to a given class of neuron. Each neuron receives afferences and efferences through various synaptic processes.

Extracellular field potentials were calculated from network activity, according to the following model (Nunez, 1981),

$$
V_{e x t}=\frac{R e}{4 \pi} \sum_{i k} \frac{I_{k, i}^{s y n}}{r_{i}}
$$

where $V_{e x t}$ is the electrical potential at a given extracellular site, $R e=230 \Omega \cdot \mathrm{cm}$ is the extracellular resistivity, $I^{s y n}$ are the scalar postsynaptic currents and $r_{i}$ is the distance between each cell and the extracellular site. For pratical reasons in the context of large network model, we did not take account of the particular cell geometry.

All simulations were implemented in a Visual-C++ code, using 4th-order Runge-Kutta integration method with a variable time step, on a Pentium IV dualprocessor, $3 \mathrm{GHz}$.

\section{RESULTS}

A. Imbalance between excitatory and inhibitory synaptic connections reveals focal epileptiform patterns similar to continuous spike-and-wave discharges

In normal conditions, without altering the network topology nor the synaptic strengths. Our model accounts correctly for mimicking non-pathological rhythms such as sleep spindles $(8-12 \mathrm{~Hz})$ and slow waves $(<1 \mathrm{~Hz})$, as confirmed by several other independent modeling studies (Wallenstein, 1994 ; Destexhe et al., 1993 ; Compte et al., 2003). Fig. 2 shows an example of local field potentials generated in conditions simulating wakefulness.

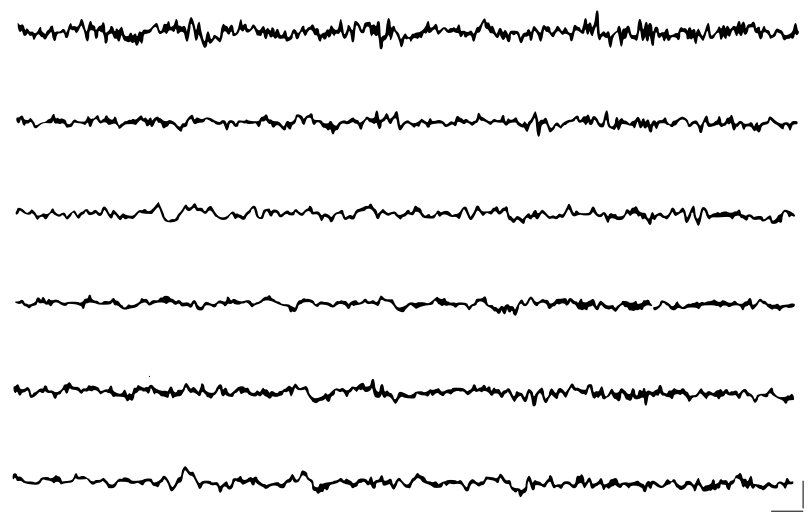

Fig. 2. Simulated extracellular field potentials mimicking heathly EEG recordings. (Calibration bar: 1s, 150mV)

By creating local anarchic connections as well as an imbalance of excitatory/inhibitory synaptic connections, our model shows local spike-and-wave discharges (cf. Fig. 3). The epileptic focus is induced by heterogeneity of parameter and local increase of glutamatergic (excitatory AMPA) synapses versus inhibitory (GABA). Keeping a normal ratio of GABA/AMPA elsewhere prevents these local discharges from propagating and generalizing during wakefulness. We can obtain these results with the presence of one or more foci with abnormal parameters values. However, synchronization properties are different in the case of multiple foci, whereas with the presence of only one focus, the number of abnormal cells seems to be critical in observing spatial propagation. Investigation of these various observations will be devoted as a perspective of the model.

\section{B. Transition to the slow-wave sleep state exacerbates} and generalizes epileptiform acitivities

We simulated the transition from the waking to the slow-wave sleep state. It is well established that 


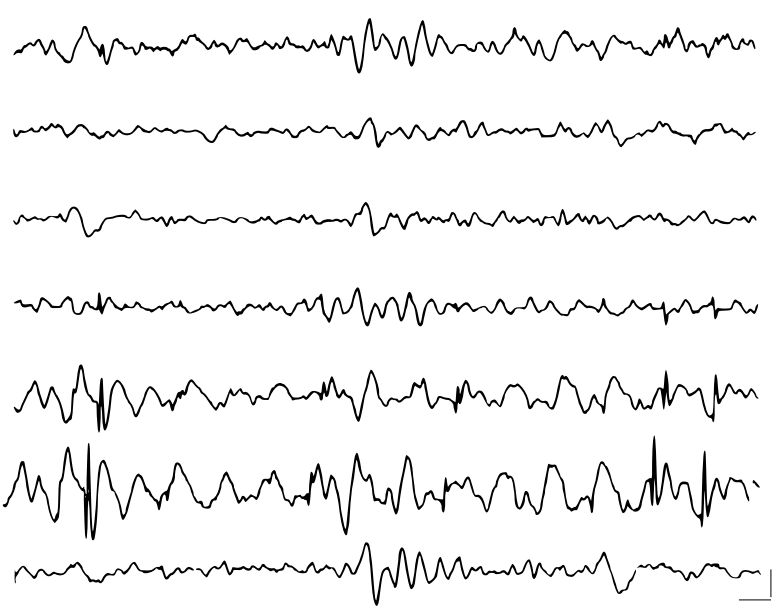

Fig. 3. Simulated extracellular field potentials mimicking EEG recordings during wakefulness. Graphs 5 and 6 show local spike-andwave seizures. No evidence of propagation. (Calibration: $1 \mathrm{~s}, 150 \mathrm{mV}$ )

the transition from wakefulness to sleep depends critically on the inactivation of ascendent activating systems (cholinergic, noradrenergic, and serotoninergic), notably a decreased cholinergic input to the cerebral cortex (McCormick, 1992). We focused on manipulations that have been linked to neuromodulatory action by acethylcholine: increase of passive, activity- and voltagedependent $\mathrm{K}^{+}$conductances, and reduced excitatory transmission.

We clearly show that the local spike-and-wave discharges are exacerbated and tend to generalize to the entire cortical network (see Fig. 4). This global synchrony is achieved as the membrane is hyperpolarized through an increase of $\mathrm{K}^{+}$conductances. As the $K^{+}$ conductances were significantly reduced, the network showed spatiotemporal coherence. We readily obtain the reverse (initial) behavior when coming back to the waking state.

\section{DISCUSSION}

One of the main symptom in LKS are aphasia and auditory agnosia. However, the relationship between EEG discharges and cognitive deficits are still unknown. The hypothesis of a causal link between epileptiform activity and these cognitive dysfunctions of language areas is still debated. The hyperactivity of glutamatergic neurons, as used in this model, may or may not be realistic. However, it would explain the suppression of the normal activity of associative cortices related to language.

A large majority of authors agree on the fact that the main EEG characteristic in LKS is consituted by highamplitude spike-waves, organized in various foci, with a predominence over temporal and parieto-occipital areas (Beaumanoir, 1992; Maquet et al., 1995).

It has been observed experimentally that drowsiness

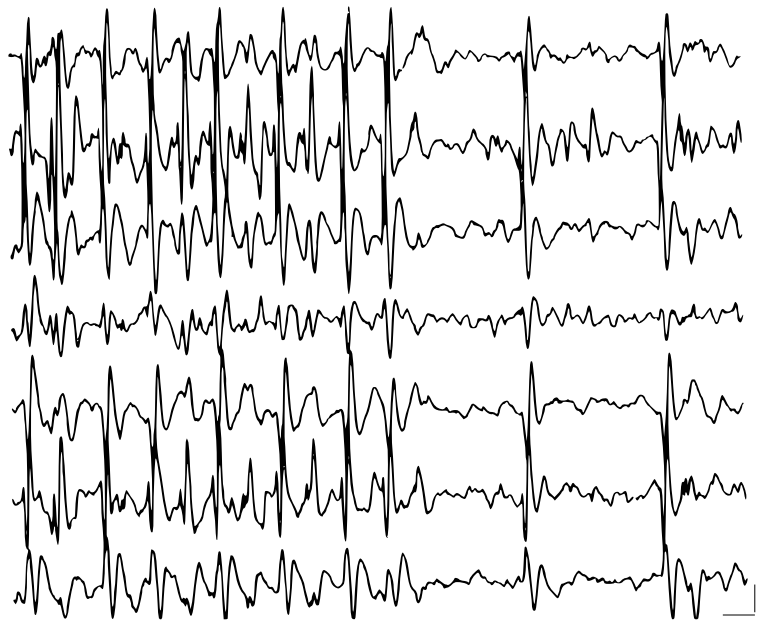

Fig. 4. Simulated extracellular field potentials mimicking EEG recordings during conditions simulating slow-wave sleep. All graphs show major spike-and-wave seizures, generalized from the initial focus. Generalized spike-and-wave discharges are observed in all cortical "areas". (Calibration: 1s, 150mV)

and sleep activate abnormalities with a large diffusion of spike-wave, especially during slow-wave sleep, which is a unique feature of LKS (Hirsch et al., 1990; Beaumanoir, 1992). From our model, we show that a local cerebral dysfunction generated using a non-common anatomical connectivity and an increase of synaptic inhibition relative to excitation lead to a focal non propagating epileptic activity, during wakefulness, which persists irrespective of the activity of the rest of the cortex. This focal cerebral dysfunction can be induced anywhere, and in multiple sites wherever anarchic connectivity and synpatic imbalance are present.

After keeping a persistent local focus of epileptiform activity where cortical glutamatergic neurons are hyperexcited and hypersynchronized, we then observed a spread and a cortical generalization of these focal discharges once the transition to deep sleep was initiated. During these phases of slow-wave sleep, extracellular field potentials are characterized by a generalization of epileptiform activity without any specific driving focus (foci).

The generalization of slow-wave discharges is related to synchronous firing in large thalamocortical populations. This firing activity uses a well described oscillatory machinery deployed during sleep (Bonjean, 2006 ; Destexhe \& Sejnowski, 2001). This explains to a certain extent the alternation between focal and secondary generalized epilepsies in the LKS and points to some basic cellular mechanisms accounting for sleeprelated activation of epileptiform activity. However, the specific causes for an unbalance between synaptic drives are beyond the scope of this investigation.

Since LKS has been observed only in children aged between 3 and 10, it has been suggested that this 
syndrome occurs only when the brain is immature, during the considerable overproduction of synapses and collaterals during the early stages of childhood (Maquet et al., 1995).

Moreover, cortical volume reduction was observed with MRI analysis in children affected by LKS, whereas they showed normal cognitive abilities before the onset of the syndrome (Takeoka et al., 2004). With this experimental observation, we think that permanent seizure activities could progressively affect the cellular population, yielding in some circumstances to neuronal death. One hypothesis is that sustained seizures can induce neuronal degeneration in the seizure focus and elsewhere within seizure pathways. If an excitotoxic mechanism underlies important components of the neuropathology of experimental seizures, it is possible that brain damage in human epilepsy may in part have a similar basis, by an early necrosis or apoptosis processes (Collins \& Olney, 1982).

\section{CONCLUSIONS AND PERSPECTIVE}

\section{A. Conclusions}

This paper proposed a realistic thalamocortical model for investigating the LKS using computational and mathematical approaches. From this model, we were able to reproduce correctly two essential characteristics of classical LKS EEG recordings, namely (1) the presence of a focus of permanent epileptic discarges during wakefulness and (2) their generalization throughout the cortex at the transition from the awake state to sleep state. From this model, we deduced a plausible cellular mechanism to account for the epileptiform activity observed experimentally by classical EEG recordings. This mechanism pointed out to the presence of a focus of permanent epileptic discharges, which generalized and spread throughout the cortex due to the transition from wakefulness to deep sleep.

\section{B. Perspective}

We hypothetized that a functional imbalance between excitatory and inhibitory drives could be responsible for the loss of cognitive abilities and paroxysmal EEG activities, although several processes, other than the ones proposed here could equally account for these phenomena. We also hypothesized that permanent epileptic seizures could be responsible for the loss of cognitive abilities and could ultimately lead to neuronal death through various processes that need to be further investigated. Simulation of neurosurgery procedures or pharmacological agents (such as the effects of benzodiazepines or other anticonvulsant drugs) are currently investigated, and would hopefully give the right orientation to theurapeutical strategies. Other interesting perspectives are related to the study of synchronization and propagation properties between multiple pathological foci.
We hope our present results and working hypotheses combined with future research will lead to a better understanding of this disorder.

\section{ACKNOWLEDGMENTS}

The authors gratefully acknowledge the contribution of the Interuniversity Attraction Poles Programme and the Belgian National Fund for Scientific Research (FNRS). The authors thanks the reviewers for helping to improve the quality of this manuscript.

\section{REFERENCES}

[1] A. Beaumanoir, The Landau-Kleffner syndrome, In: Epileptic syndromes in infancy, childhood, and adolescence, 2nd ed. Chichester, 231-43, 1992.

[2] M. Bonjean, Towards a realistic model of the thalamocortical circuitry, MD. Thesis, University of Liege, 2006.

[3] R.C. Collins and J.W. Olney, Focal cortical seizures cause distant thalamic lesions, Science, 218:177-179, 1982.

[4] A. Compte, M.V. Sanchez-Vives, D.A. McCormick, X-J. Wang, Cellular and network mechanisms of slow oscillatory activity and wave propagations in a cortical network model, J. Neurophysiol., 89:2707-25, 2003.

[5] A. Destexhe, D.A. McCormick, T.J. Sejnowski, A model for 8$10 \mathrm{~Hz}$ spindling in interconnected thalamic relay and reticularis neurons, Biophys. J., 65:2474-8, 1993.

[6] A. Destexhe and T.J. Sejnowski, Thalamocortical assemblies, Oxford Univ. Press, 2001.

[7] E. Hirsch, C. Marescaux, P. Maquet, M.N. Metz-Lutz, M. Kiesmann, E. Salmon, et al., Landau-Kleffner syndrome: a clinical and EEG study of five cases, Epilepsia, 31:756-67, 1990.

[8] A.L. Hodgkin, and A.F. Huxley, A quantitative description of membrane current and its application to conduction and excitation in nerve, J. Physiol, 117:500-544, 1952.

[9] J. Laidlaw, A. Richens, and D. Chadwick, A Textbook of Epilepsy, 4th ed., Churchull Livingstone, 1993.

[10] W.M. Landau and F.R. Kleffner, Syndrome of acquired aphasia with convulsive disorder in children, Neurology, 7:523-30, 1957.

[11] J.F. Mantovani and W.M. Landau, Acquired aphasia with convulsive disorder: course and prognosis, Neurology, 30:524-9, 1980.

[12] P. Maquet, E. Hirsch, D. Dive, E. Salmon, C. Marescaux, and G. Franck, Cerebral glucose utilization during sleep in LandauKleffner syndrome: a PET study, Epilepsia, 31:778-83, 1990.

[13] P. Maquet, E. Hirsch, M.N. Metz-Lutz, J. Motte, D. Dive, C. Marescaux, and G. Franck, Regional cerebral glucose metabolism in children with deterioration of one or more cognitive functions and continuous spike-and-wave discharges during sleep, Brain, 118:1497-1520, 1995.

[14] D.A. McCormick, Neurotransmitter actions in the thalamus and cerebral cortex and their role in neuromodulation of thalamocortical activity, Prog. Neurobiol., 39:337-88, 1992.

[15] P.L. Nunez, Electric fields of the brain. The neurophysics of EEG, Oxford Univ. Press, 1981.

[16] P.F. Pinsky, and J. Rinzel, Intrinsic and network rhythmogenesis in a reduced Traub model for CA3 neurons, J. Comput. Neurosci., 1:39-60, 1994.

[17] G. Shepherd, The synaptic organization of the brain, 5th ed., Oxford Univ. Press, 2003.

[18] C. Stafstrom, Epilepsy: a review of selected clinical syndromes and advances in basic science, J. Cereb. Blood Flow Metab., 26(8):983-1004, 2006.

[19] M. Takeoka, J.J. Riviello, F.H. Duffy, F. Kim, D.N. Kennedy, N. Makris, V.S. Caviness, and G.L. Holmes, Bilateral volume reduction of the superior temporal areas in Landau-Kleffner syndrome, Neurology, 63:1289-92, 2004.

[20] G.V. Wallenstein, A model of the electrophysiological properties of nucleus reticularis thalami neurons, Biophys. J., 66:978-88, 1994.

[21] X-J. Wang, Calcium coding and adaptive temporal computation in cortical pyramidal neurons, J. Neurophysiol., 79:1549-66, 1998. 